Article

\title{
Sacrificial Template-Based Synthesis of Unified Hollow Porous Palladium Nanospheres for Formic Acid Electro-Oxidation
}

\author{
Xiaoyu Qiu, Hanyue Zhang, Yuxuan Dai, Fengqi Zhang, Peishan Wu, Pin Wu and Yawen Tang* \\ Jiangsu key laboratory of Key Laboratory of New Power Batteries, Jiangsu Collaborative Innovation \\ Center of Biomedical Functional Materials, School of Chemistry and Materials Science, Nanjing \\ Normal University, Nanjing 210023, China; E-Mails: qxy18251813615@163.com (X.Q.); \\ lovelybabyzhang@sina.com (H.Z.); dyx12138@sina.com (Y.D.); zhangfengqi@163.com (F.Z.); \\ wupeishan@163.com (P.W.); zjuwuping@njnu.edu.cn (P.W.) \\ * Author to whom correspondence should be addressed; E-Mail: tangyawen@njnu.edu.cn; \\ Tel.: +86-25-85891651.
}

Academic Editor: Minhua Shao

Received: 1 April 2015 / Accepted: 16 June 2015 / Published: 23 June 2015

\begin{abstract}
Large scale syntheses of uniform metal nanoparticles with hollow porous structure have attracted much attention owning to their high surface area, abundant active sites and relatively efficient catalytic activity. Herein, we report a general method to synthesize hollow porous $\mathrm{Pd}$ nanospheres ( $\mathrm{Pd} \mathrm{HPNSs}$ ) by templating sacrificial $\mathrm{SiO}_{2}$ nanoparticles with the assistance of polyallylamine hydrochloride (PAH) through layer-by-layer self-assembly. The chemically inert PAH is acting as an efficient stabilizer and complex agent to control the synthesis of Pd HPNSs, probably accounting for its long aliphatic alkyl chains, excellent coordination capability and good hydrophilic property. The physicochemical properties of Pd HPNSs are thoroughly characterized by various techniques, such as transmission electron microscopy, X-ray diffraction, X-ray photoelectron spectroscopy. The growth mechanism of Pd HPNSs is studied based on the analysis of diverse experimental observations. The as-prepared Pd HPNSs exhibit clearly enhanced electrocatalytic activity and durability for the formic oxidation reaction (FAOR) in acid medium compared with commercial Pd black.
\end{abstract}

Keywords: hollow porous $\mathrm{Pd}$ nanospheres; polyallylamine hydrochloride; sacrificial $\mathrm{SiO}_{2}$ nanoparticles; formic oxidation reaction 


\section{Introduction}

Palladium ( $\mathrm{Pd}$ ) plays an important role in a variety of chemical reactions [1-4], such as serving as an efficient electrocatalyst towards formic acid oxidation reaction (FAOR) in direct formic acid fuel cells (DFAFCs) [5-7]. Compared to Pt catalysts, Pd has lower cost with relatively higher abundance, emerging as a promise substitute of Pt especially in the development of DFAFCs [8,9]. Obviously, the morphology of $\mathrm{Pd}$ nanocrystals powerfully influences their electrocatalytic activity and stability because of structural effects. For example, G. Fu and his group have successfully synthesized Pd nanoparticles with nanocubes and icosahedra structure [10,11]. For hollow Pd nanosphere with porous structure, the advantage is prominent, as follows [12]: (1) The hollow porous structure gives birth to a combination of high specific surface area, low mass-density and enhanced reaction kinetics due to extra confined internal reaction space $[13,14]$. (2) Such hollow porous nanostructures not only supply abundant active sites owning to ample edges and corners, but also improve the mass transfer promoting the electrocatalytic activity obviously [15]. (3) Homogeneous hollow porous nanocrystals are less vulnerable to Ostwald ripening, dissolution and aggregation, thus probably restraining the attenuation of the catalyst activity [16].

In this work, positively charged colloidal modified silica serve as a sacrificial template to synthesize the hollow porous Pd nanospheres (Pd HPNSs). The layer-by-layer (LBL) approach is used to functionalize the $\mathrm{SiO}_{2}$ template with evenly-spread positive charges around the outside surface (PAH/PSS/PAH-SiO 2 ) [17]. The positively charged silica could powerfully adsorb the $\mathrm{PdCl}_{4}{ }^{2-}$ and $\mathrm{BH}_{4}^{-}$, which are negatively charged metal ions, resulting in the reduction reaction mostly taking place on the surface of the $\mathrm{SiO}_{2}$. The outermost PAH not only functions as polyelectrolyte to strongly adsorb negatively charged precursors and reductants, but also serves as a stabilizer and complex agent to effectively avoid the aggregation of the Pd nanoparticle owning to its long aliphatic alkyl chain and excellent hydrophilic property. $\mathrm{NaBH}_{4}$ is chosen as a strong reductant to rapidly form the uniform Pd nanospheres. After removing the $\mathrm{SiO}_{2}$ sacrificial template, the morphology of the outermost $\mathrm{Pd}$ nanospheres is maintained to obtain the hollow porous nanostructure. The as-prepared Pd HPNSs exhibit observably enhanced electrocatalytic activity and durability for FAOR compared with commercial Pd black [18-21].

\section{Results and Discussion}

\subsection{Physicochemical Characterization of Pd HPNSs}

Figure 1 shows the schematic representation for the synthesis of Pd HPNSs. $\mathrm{SiO}_{2}$ nanosphere is chosen as a sacrificial template owning to its hydrophilia, mechanical stability, and controllability of size and morphology [22-25]. After the layer-by-layer self-assembly of charged polyelectrolyte on $\mathrm{SiO}_{2}$ template, $\mathrm{PdCl}_{4}{ }^{2-}$ can be uniformly adsorbed owning to electrostatic attraction, and then be in-situ reduced by $\mathrm{NaBH}_{4}$, mostly taking place on the surface of the $\mathrm{SiO}_{2}$. After removing the $\mathrm{SiO}_{2}$ sacrificial template, the hollow porous nanospheres structure of Pd HPNSs can be obtained and remains unchanged even after a long time storage. Photographs are taken to prove the powerful adsorption of PAH/PSS/PAH-SiO 2 in solution (Figure 2). After two-phase centrifuging, the precipitate of modified solid $\mathrm{SiO}_{2}$ nanoparticles in white, and in transparent supernatant liquor, is observed 
(Figure 2A). In contrast, when $\mathrm{PdCl}_{4}{ }^{2-}$ was dropped into the mixture, forming a well-distributed mixture solution, the precipitate turns $t$ yellow and the supernatant liquor stays transparent, indicating the completely adsorption of $\mathrm{PdCl}_{4}{ }^{2-}$. This will make sure the follow-up reduction reaction takes place on the surface of $\mathrm{SiO}_{2}$ templates (Figure 2B).

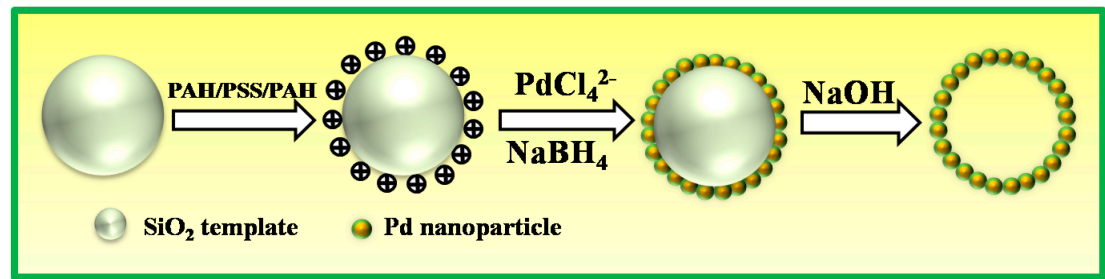

Figure 1. Schematic illustration of the fabrication procedure to produce hollow porous $\mathrm{Pd}$ nanospheres (Pd HPNSs).

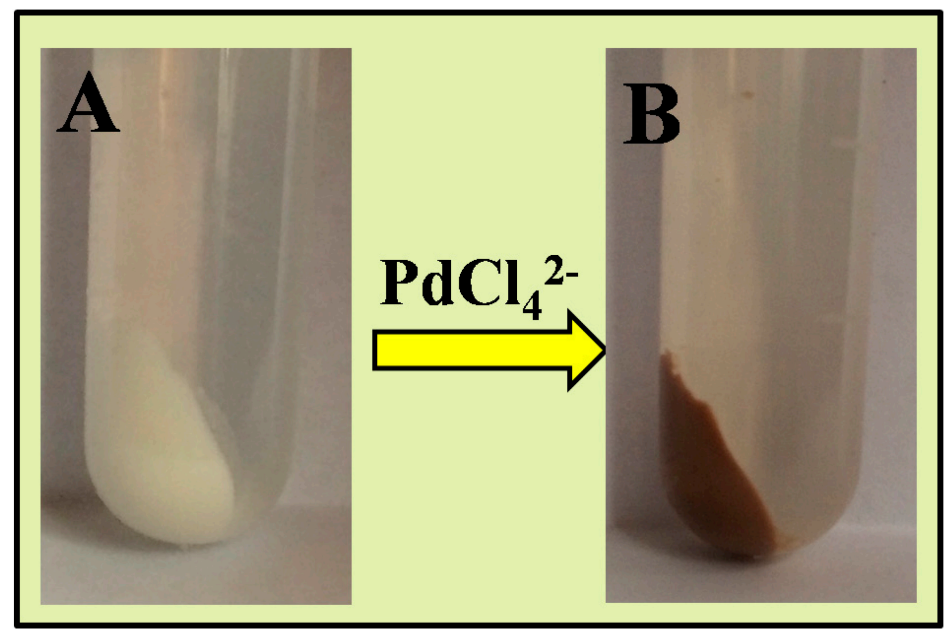

Figure 2. Photographs of (A) $\mathrm{PAH} / \mathrm{PSS} / \mathrm{PAH}$-modified $\mathrm{SiO}_{2}$ solution after centrifuging and (B) Mixture of $\mathrm{PdCl}_{4}{ }^{2-}$ and $\mathrm{PAH} / \mathrm{PSS} / \mathrm{PAH}-$ modified $\mathrm{SiO}_{2}$ solution after centrifuging.

The morphology of the as-prepared nanoparticles at different stages is investigated by SEM and TEM. Figure 3 represents the SEM/TEM images of $\mathrm{SiO}_{2}, \mathrm{Pd}-\mathrm{SiO}_{2}$ and $\mathrm{Pd} \mathrm{HPNSs}$. As shown in Figure 3A,B, the $\mathrm{SiO}_{2}$ particles possess a similar size of ca. $200 \mathrm{~nm}$. The images of $\mathrm{Pd}-\mathrm{SiO}_{2}$ as shown in Figure 3C,D confirm that when $\mathrm{Pd}$ was sequentially deposited on the surface of polyelectrolyte-modified $\mathrm{SiO}_{2}$ nanoparticles, the surface becomes less smooth and rougher without obvious morphology change. No individual $\mathrm{Pd}$ nanoparticles can be found except on the surface of $\mathrm{SiO}_{2}$ templates (shown in the yellow area in Figure 3D), demonstrating the in-situ formation of Pd nanoparticles. After removing the $\mathrm{SiO}_{2}$ template, hollow porous nanospheres structure of Pd HPNSs can be obtained (Figure 3E, F). The yellow arrow highlights one of the broken hollow nanospheres, from which it is clear that the inner space in the as-prepared Pd HPNSs was vacant, indicating that the template of $\mathrm{SiO}_{2}$ was removed, leading to a hollow structure. The TEM image of Pd HPNSs in Figure 3F also demonstrates that the structural integrity of most produced Pd HPNSs with narrow distribution is well maintained even after sonication for a long period of time, indicating superior mechanical properties of the as-prepared Pd HPNSs. 


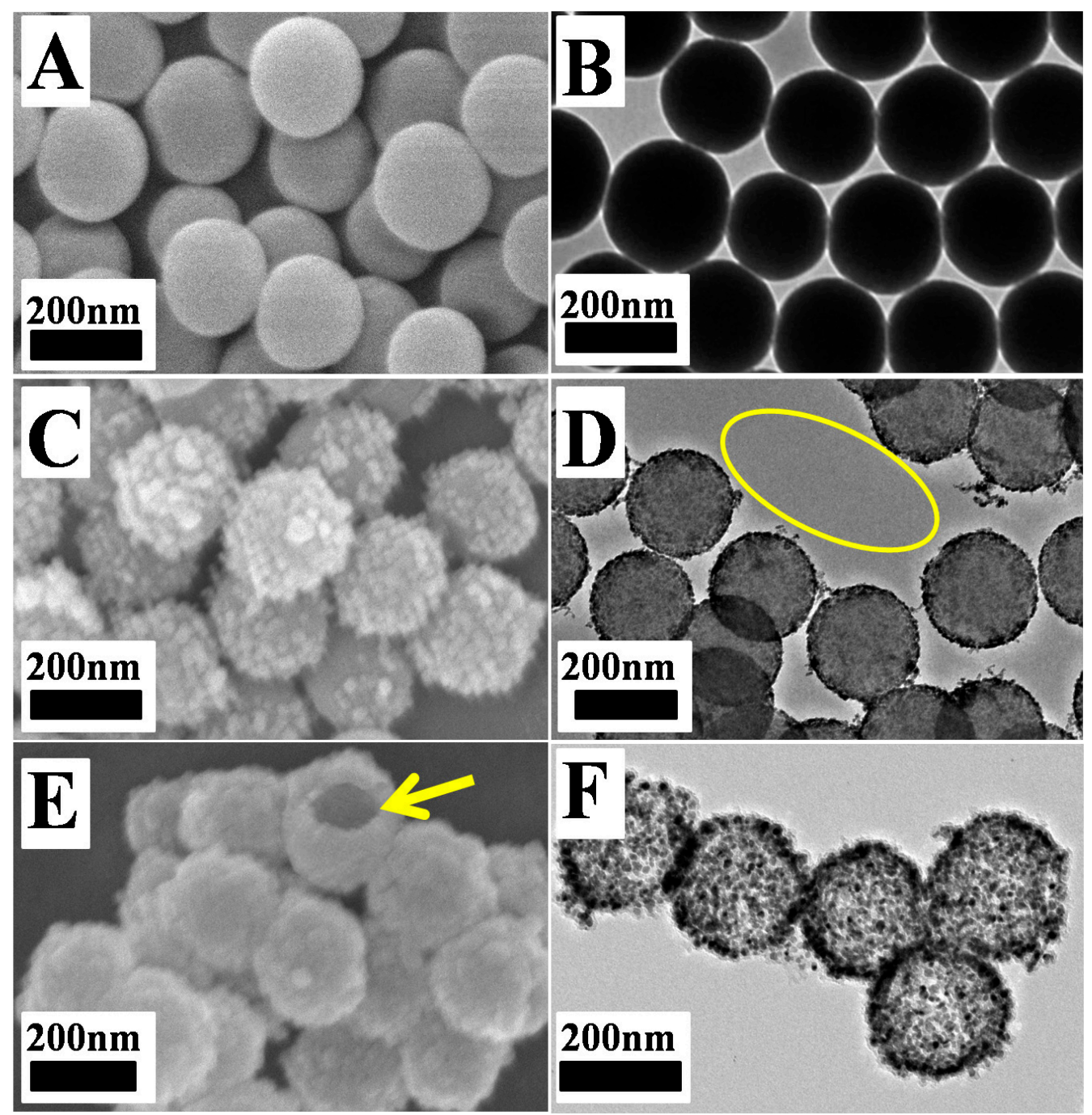

Figure 3. SEM images (A, C and $\mathbf{E})$ and large-area TEM images $(\mathbf{B}, \mathbf{D}$ and $\mathbf{F})$ of $\mathrm{SiO}_{2}(\mathbf{A}$ and $\mathbf{B}), \mathrm{Pd}_{-} \mathrm{SiO}_{2}(\mathbf{C}$ and $\mathbf{D})$ and Pd HPNSs $(\mathbf{E}$ and $\mathbf{F})$.

Representative large-area TEM image (Figure 4A) and middle-resolution TEM image (Figure 4B) of an individual Pd HPNS clearly show the inner and outer surfaces of the hollow porous spheres, displaying the interconnection through shared outside surface and good dispersibility of the $\mathrm{Pd}$ nanoparticles $(\sim 4-5 \mathrm{~nm})$. Further magnified HRTEM image (Figure 4C) from yellow region of Figure 4B shows an interplanar spacing with $0.225 \mathrm{~nm}$, which is close to the $\{111\}$ lattice spacing of face-centered cubic (fcc) Pd. The selected-area electron diffraction (SAED) image of an individual Pd HPNS shows diffraction rings corresponding to various facets of face-centered cubic (fcc) (inset in Figure 4C), demonstrating that Pd HPNSs have polycrystalline structure. To observe more clearly about the distribution of Pd nanoparticles, the EDS mapping (Figure 4D) are performed. The resulting patterns show the uniform distribution of Pd throughout the whole Pd HPNS, a strong evidence for the formation of Pd HPNSs as well. Then, the product of different stages is further investigated by XRD (Figure 4E). XRD pattern demonstrates that $\mathrm{SiO}_{2}-\mathrm{Pd}$ nanospheres have both diffraction peaks of $\mathrm{SiO}_{2}$ and $\mathrm{Pd}$, while the diffraction peak of $\mathrm{SiO}_{2}$ disappears from the XRD pattern of Pd HPNSs, demonstrating the completely remove of $\mathrm{SiO}_{2}$ template. What's more, from XRD pattern of the Pd 
HPNSs, fcc structure can be identified as the diffraction peaks of the products are located at the same position as those of pure fcc Pd (PDF\#46-1043) crystal phases. The average particle size of the small Pd nanoparticles is $4.3 \mathrm{~nm}$ calculated from the peak width of the Pd (111) diffraction according to the Scherrer's equation. It is well known that the near-surface composition plays a critical role on the electrocatalytic behavior of noble-metal catalysts. Thus, the near-surface compositional feature of the Pd HPNSs is examined by XPS (Figure 4F). As observed, no obvious signals of Si and $\mathrm{O}$ can be observed and the signals of $\mathrm{Pd}$ are strong. Further, the $\mathrm{Pd} 3 \mathrm{~d}$ signals are deconvoluted into two components: Pd 3d $3 / 2(340.6 \mathrm{eV}), \mathrm{Pd} 3 \mathrm{~d}_{5 / 2}(335.2 \mathrm{eV})$ and $\mathrm{Pd} 3 \mathrm{~d}_{3 / 2}(341.7 \mathrm{eV}), \mathrm{Pd} 3 \mathrm{~d}_{5 / 2}(336.3 \mathrm{eV})$, which are assigned to $\mathrm{Pd}^{0}$ and $\mathrm{Pd}^{\mathrm{II}}$ species, respectively. By measuring the relative peak areas, the percentage of $\mathrm{Pd}^{0}$ species in the Pd HPNSs is calculated to be $92.1 \%\left(\mathrm{Pd}^{0} /\left(\mathrm{Pd}^{0}+\mathrm{Pd}^{\mathrm{II}}\right)\right)$, much higher than the reported value of $\mathrm{Pd}$ nanoparticles.
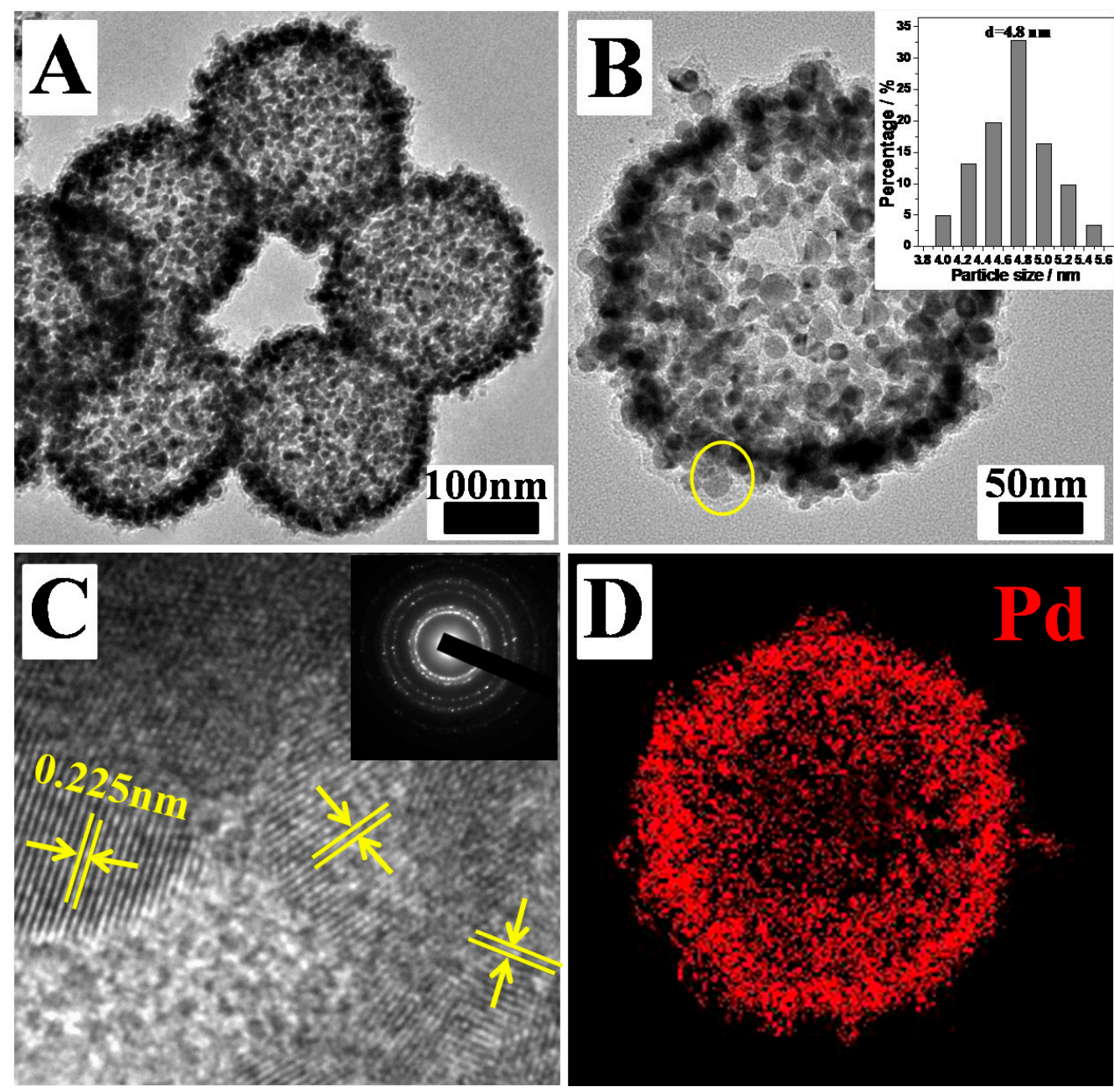

Figure 4. Cont. 


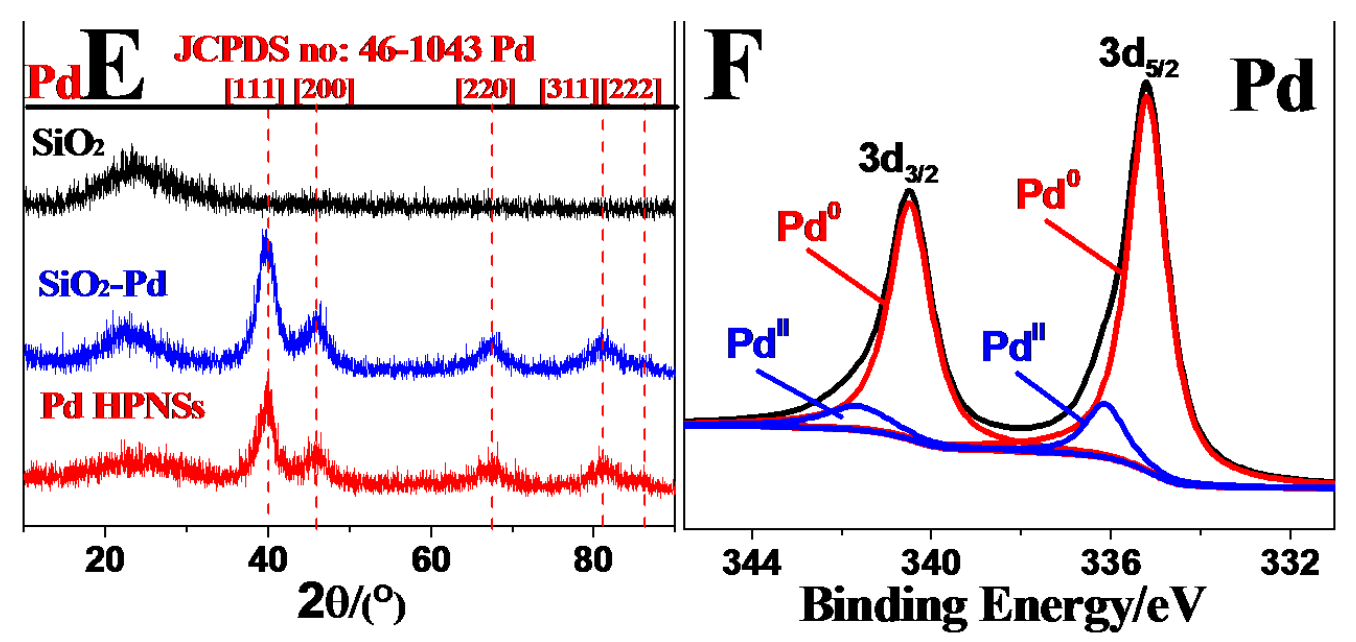

Figure 4. (A) Representative large-area transmission electron microscopy (TEM) image of Pd HPNSs. (B) Middle-resolution TEM image of an individual Pd HPNSs. Insert: histograms of the particle size distribution (C) Magnified HRTEM images recorded from Figure 4B. Inset in Fig. C: selected-area electron diffraction (SAED) pattern of an individual Pd HPNSs. (D) EDX elemental mapping patterns of Pd HPNSs. (E) X-ray diffraction (XRD) patterns of the $\mathrm{SiO}_{2}$ templates, $\mathrm{SiO}_{2}-\mathrm{Pd}$ nanospheres, and Pd HPNSs. (F) XPS spectra of Pd HPNSs in the Pd $3 d$ regions.

\subsection{Electrocatalytic Tests}

The electrocatalytic properties of Pd HPNSs for the FAOR were examined and compared with commercial Pd black. The electrochemically active surface areas (ECSA) of Pd HPNSs and commercial Pd black were measured by CO-stripping measurements (Figure 5A). It is observed that the ECSA of Pd HPNSs on glassy carbon electrode is $1.4 \mathrm{~cm}^{2}, 1.20$ times higher than that of the commercial Pd black $\left(1.2 \mathrm{~cm}^{2}\right)$, which can be ascribed to the small particle size and hollow porous structure of Pd HPNSs. The mass-normalized cyclic voltammogram shows the FAOR peak potential on Pd HPNSs negatively shifts $110 \mathrm{mV}$ compared to that of commercial Pd black in the forward scan (Figure 5B). Moreover, FAOR peak current on Pd HPNSs reaches a value of $203.2 \mathrm{~mA} \mathrm{mg}^{-1}$, which is about 1.4 times higher than that of commercial Pd black $\left(140.6 \mathrm{~mA} \mathrm{mg}^{-1}\right)$. It is well-known that the specific kinetic activity (normalized to the ECSA) of a catalyst can effectively evaluate the actual value of the intrinsic activity. Further ECSA-normalized cyclic voltammograms show FAOR peak current on Pd HPNSs is 1.2 times higher than that on commercial Pd black (Figure 5C). The lower FAOR onset oxidation potential and peak potential, the bigger mass-activity and specific activity demonstrate that Pd HPNSs have good electrocatalytic performance for the FAOR, holding promise as potentially practical electrocatalysts for the FAOR. The improved electrocatalytic performance may mainly originate from the unique hollow porous structure. The electrochemical stability of Pd HPNSs for the FAOR is investigated by chronoamperometry at $0.1 \mathrm{~V}$ potential (Figure 5D). FAOR current on Pd HPNSs is higher than commercial Pd black during the whole reaction process. At $3000 \mathrm{~s}$, formic oxidation currents on the Pd HPNSs and Pd black decrease to $30.76 \%$ and $5.15 \%$ of their initial values (taken at $20 \mathrm{~s}$ to avoid the contribution of the double-layer discharge and hydrogen adsorption), indicating Pd HPNSs have superior durability for the FAOR. 


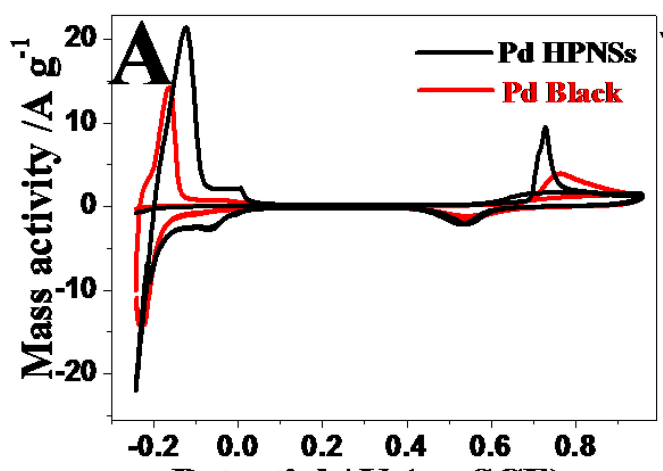

Potential / V (vs. SCE)

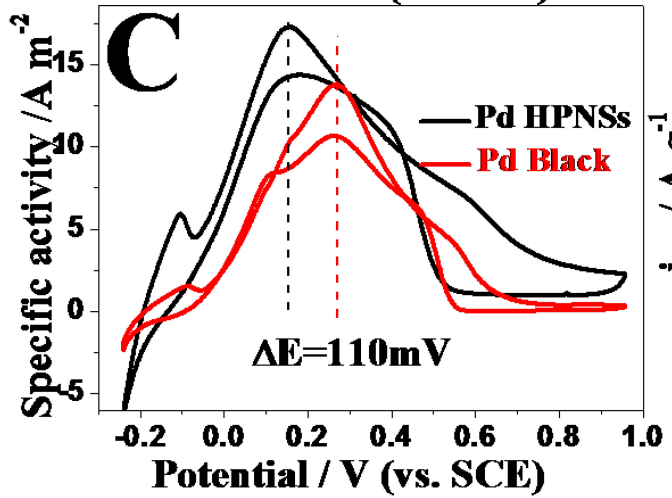

Figure 5. (A) CV curves of the Pd HPNSs and Pd black in $\mathrm{N}_{2}$-saturated $0.5 \mathrm{M} \mathrm{H}_{2} \mathrm{SO}_{4}$ solution at $50 \mathrm{mV} \mathrm{s}^{-1}$. (B) The metal mass-normalized cyclic voltammograms for the Pd HPNSs and Pd black in solution of $0.5 \mathrm{M} \mathrm{HCOOH}+0.5 \mathrm{M} \mathrm{H}_{2} \mathrm{SO}_{4}$ at a scan rate of $50 \mathrm{mV} \mathrm{s}^{-1}$. (C) ESCA-normalized cyclic voltammograms of Pd HPNSs and Pd black in solution of 0.5 $\mathrm{M} \mathrm{HCOOH}+0.5 \mathrm{M} \mathrm{H}_{2} \mathrm{SO}_{4}$ at a scan rate of $50 \mathrm{mV} \mathrm{s}^{-1}$. (D) Chronoamperometry curves for the Pd HPNSs and Pd black in solution of $0.5 \mathrm{M} \mathrm{HCOOH}+0.5 \mathrm{M} \mathrm{H}_{2} \mathrm{SO}_{4}$ for $3000 \mathrm{~s}$ at $0.1 \mathrm{~V}$ potential.

\section{Experimental Section}

\subsection{Reagents and Chemicals}

Poly (allylamine hydrochloride) (PAH, weight-average molecular weight 150 000) was supplied from Nitto Boseki Co., L t d. (Tokyo, Japan). Poly (sodium 4-styrenesulfonate) (PSS, $M_{\mathrm{w}}<700000 \mathrm{Da}$ ), was purchased from Alfa. Aesar Co. Ltd. (Tokyo, Japan). Potassium tetrachloropalladite(II) $\left(\mathrm{K}_{2} \mathrm{PdCl}_{4}\right)$ and sodium borohydride $\left(\mathrm{NaBH}_{4}\right)$ were purchased from Sinopharm Chemical Reagent Co., Ltd (Shanghai, China). Commercial Pd black were purchased from Johnson Matthey Corporation (London, UK). All the reagents were of analytical reagent grade and used without further purification.

\subsection{Synthesis of Hollow porous Pd Nanospheres (Pd HPNSs)}

$\mathrm{SiO}_{2}$ sphere templates with a diameter of ca. $200 \mathrm{~nm}$ were synthesized by tetraethyl orthosilicate (TEOS) hydrolyzation in alkaline condition. Typically, $100 \mathrm{~mL}$ ethanol, $6 \mathrm{~mL}$ ammonium hydroxide, $6 \mathrm{~mL} \mathrm{H} \mathrm{H}_{2} \mathrm{O}, 3 \mathrm{~mL}$ TEOS were mixed and mechanically stirred for $5 \mathrm{~h}$. After the reaction, the obtained $\mathrm{SiO}_{2}$ templates were separated by centrifugation at $8500 \mathrm{rpm}$ for $5 \mathrm{~min}$, washed several times with 
water, and then dried at $60{ }^{\circ} \mathrm{C}$ for $5 \mathrm{~h}$ in a vacuum dryer. Then, the positively charged modified $\mathrm{SiO}_{2}$ was prepared through a layer-by-layer self-assembly method via electrostatic attraction between charged species. $\mathrm{SiO}_{2}$ templates were treated with Poly (allylamine hydrochloride) (PAH) and poly (sodium 4-styrenesulfonate) (PSS) in sequence, yielding positively charged PAH/PSS/PAH-modified $\mathrm{SiO}_{2}$ templates [22]. To obtain the Pd HPNSs, $30 \mathrm{mg} \mathrm{PAH} / \mathrm{PSS} / \mathrm{PAH}$-modified $\mathrm{SiO}_{2}$ and $30 \mathrm{mg}$ $\mathrm{K}_{2} \mathrm{PdCl}_{4}$ were added into $40 \mathrm{~mL}$ water and then sonicated for $30 \mathrm{~min}$. After sonication, $10 \mathrm{mg} \mathrm{NaBH}$ was added into the mixture and mechanically stirred for 1 hour at room temperature to obtain $\mathrm{SiO}_{2}-\mathrm{Pd}$ nanospheres. Then, $15 \mathrm{~mL} 2 \mathrm{M} \mathrm{NaOH}$ solution was used to remove the $\mathrm{SiO}_{2}$ sacrificial template, followed by centrifuging, washing with distilled water and ethanol, and then dried in a vacuum oven at $50{ }^{\circ} \mathrm{C}$ to obtain the Pd HPNSs.

\subsection{Electrochemical Instrument}

All electrochemical experiments were measured with a $\mathrm{CHI} 660 \mathrm{C}$ electrochemical analyzer $(\mathrm{CH}$ Instruments, Shanghai, Chenghua Co.). All electrochemical measurements were carried out at $30 \pm 1{ }^{\circ} \mathrm{C}$. A standard three-electrode system (consisted of a saturated calomel reference electrode (SCE), a catalyst modified glassy carbon electrode as the working electrode, and a platinum wire as the auxiliary electrode) was used to test all electrochemical experiments. An evenly distributed suspension of catalyst was prepared by ultrasonic the mixture of $10 \mathrm{mg}$ catalyst and $5 \mathrm{~mL} \mathrm{H}_{2} \mathrm{O}$ for $30 \mathrm{~min}$, and $6 \mu \mathrm{L}$ of the resulting suspension was loaded on the surface of the glassy carbon electrode ( $3 \mathrm{~mm}$ diameter, $0.07 \mathrm{~cm}^{2}$ ). Thus, the working electrode was obtained, and the total mass loading of catalyst on the electrode was about $12 \mu \mathrm{g}$. We used the same amount of total metal of Pd HPNSs and commercial Pd Black to make a comparison. Throughout the cyclic voltammetry experiment, cyclic voltammetry tests were performed in $\mathrm{N}_{2}$-saturated $0.5 \mathrm{M} \mathrm{H}_{2} \mathrm{SO}_{4}$ solution with or without $0.5 \mathrm{M}$ $\mathrm{HCOOH}$ at a scan rate of $50 \mathrm{mV} \mathrm{s}^{-1}$. Chronoamperometry curves were obtained in $\mathrm{N}_{2}$-saturated $0.5 \mathrm{M}$ $\mathrm{HCOOH}+0.5 \mathrm{M} \mathrm{H}_{2} \mathrm{SO}_{4}$ mixture solution for $3000 \mathrm{~s}$ at $0.1 \mathrm{~V}$ applied potential.

\subsection{Instruments}

Transmission electron microscopy (TEM) images were surveyed from a JEOL JEM-2100F transmission electron microscopy operated at an accelerating voltage of $200 \mathrm{kV}$. X-ray diffraction (XRD) patterns were obtained from a Model D/max-rC X-ray diffractometer by using $\mathrm{Cu} \mathrm{K} \alpha$ radiation source $(\lambda=1.5406 \AA$ ), operating at $40 \mathrm{kV}$ and $100 \mathrm{~mA}$. X-ray photoelectron spectroscopy (XPS) measurements were carried out on a Thermo VG Scientific ESCALAB 250 spectrometer with an Al $\mathrm{K} \alpha$ radiator. The vacuum in the analysis chamber was maintained at about $10^{-9} \mathrm{mbar}$ and the binding energy was calibrated by means of the $\mathrm{C} 1 \mathrm{~s}$ peak energy of $284.6 \mathrm{eV}$.

\section{Conclusions}

In summary, the hollow porous Pd nanospheres with high surface area, low mass-density and abundant active sites are synthesized by a sacrificial template method over PAH. The layer-by-layer (LBL) approach is used to modify the $\mathrm{SiO}_{2}$ template in order to make it positively charged. PAH not only functions as polyelectrolyte to strongly adsorb negatively charged precursors, ensuring the 
reduction reaction completely take place on the surface of the $\mathrm{SiO}_{2}$, but also serves as stabilizer and complex agent to effectively avoid the aggregation and collapse of the Pd HPNSs owning to its coordination capability, good hydrophilic property, and high chemical stability. Undoubtedly, this method is more promising from an environmental standpoint, adding advantage over the use of high-temperature reaction and toxic organic solvents. Electrochemical measurements demonstrate that Pd HPNSs exhibit superior electrocatalytic activity and long-term durability compared to commercial Pd black. Thus, the superior electrocatalytic performance of Pd HPNSs provides a promising support for good electrocatalyst in DFAFC applications.

\section{Acknowledgments}

The authors are grateful for the financial support of NSFC (21376122 and 21273116), United Fund of NSFC and Yunnan Province (U1137602), the National Basic Research Program of China (973 Program, 2012CB215500), Postgraduate Research and Innovation Project in Jiangsu Province (CXLX13-369), and a project funded by the Priority Academic Program Development of Jiangsu Higher Education Institutions, and National and Local Joint Engineering Research Centre of Biomedical Functional Materials.

\section{Author Contributions}

Xiaoyu Qiu prepared the samples, performed the experiment and wrote manuscript. Hanyue Zhang and Yuxuan Dai supported the experiments. Fengqi Zhang and Peishan Wu carried out the XRD analysis. Ping $\mathrm{Wu}$ and Yawen Tang revised the final version of paper.

\section{Conflicts of Interest}

The authors declare no conflict of interest.

\section{References}

1. Nelson, N.; Manzano, J.; Sadow, A.; Overbury, S.; Slowing, I. Selective Hydrogenation of Phenol Catalyzed by Palladium on High-Surface-Area Ceria at Room Temperature and Ambient Pressure. ACS Catal. 2015, 5, 2051-2061

2. Cervantes, C.; Alamo, M.; Garcia, J. Hydrogenation of Biomass-Derived Levulinic Acid into $\gamma$-Valerolactone Catalyzed by Palladium Complexes. ACS Catal. 2015, 5, 1424-1431.

3. Zhu, F.; Wang, Z. Palladium-Catalyzed Coupling of Azoles or Thiazoles with Aryl Thioethers via C-H/C-S Activation. Org. Lett. 2015, 2, doi:10.1021/acsarglett5b00510.

4. Zhao, G.; Chen, C.; Yue, Y.; Yu, Y.; Peng, J. Palladium(II)-Catalyzed Sequential C-H Arylation/Aerobic Oxidative $\mathrm{C}-\mathrm{H}$ Amination: One-Pot Synthesis of BenzimidazoleFusedPhenanthridines from 2-Arylbenzimidazoles and Aryl Halides JOC. J. Org. Chem. 2014, 11, doi:10.1021/ja502632b.

5. Yang, S.; Shen, C.; Lu, X.; Tong, H.; Zhu, J.; Zhang, X.; Gao, H. Preparation and electrochemistry of graphene nanosheets-multiwalled carbon nanotubes hybrid nanomaterials as Pd electrocatalyst support for formic acid oxidation. Electrochim. Acta 2012, 62, 242-249. 
6. Yang, S.; Dong, J.; Yao, Z.; Shen, C.; Shi, X.; Tian, Y.; Lin, S.; Zhang, X. One-Pot Synthesis of Graphene-Supported Monodisperse Pd Nanoparticles as Catalyst for Formic Acid Electro-oxidation. Sci. Rep. 2014, 3, doi: 10.1038/srep04501.

7. Wang, Y.; Liu, H.; Wang, L.; Wang, H.; Du, X.; Wang, F.; Qi, T.; Lee, J.; Wang, X. Pd catalyst supported on a chitosan-functionalized large-area 3D reduced graphene oxide for formic acid electrooxidation reaction. J. Mater. Chem. A 2013, 4, doi:10.1039/c3ta10214a.

8. Chang, J.; Sun, X.; Feng, L.; Xing, W.; Qin, X.; Shao, G. Effect of nitrogen-doped acetylene carbon black supported Pd nanocatalyst on formic acid electrooxidation. J. Power Sources 2013, 239, 94-102.

9. Bai, Z.; Yan, H.; Wang, F.; Yang, L.; Jiang, K. Electrooxidation of formic acid catalyzed by Pd Nanoparticles supported on multi-walled carbonnanotubes with sodium oxalate. Ionics 2013, 19, 543-548.

10. Fu, G.; Jiang, X.; Tao, L.; Chen, Y.; Lin, J.; Zhou, Y.; Tang, Y.; Lu T. Polyallylamine Functionalized Palladium Icosahedra: One-Pot Water-Based Synthesis and Their Superior Electrocatalytic Activity and Ethanol Tolerant Ability in Alkaline Media. Langmuir 2013, 29 , 4413-4420.

11. Fu, G.; Wu, K.; Jiang, X.; Tao, L.; Chen, Y.; Lin, J.; Zhou, Y.; Wei, S.; Tang, Y.; Lu, T.; Xia, X. Polyallylamine-directed green synthesis of platinum nanocubes. Shape and electronic effect codependent enhanced electrocatalytic activity. Phys. Chem. Chem. Phys. 2013, 15, 3793-3802.

12. Yang, Y.; Wang, F.; Yang, Q.; Hu, Y.; Yan, H.; Chen, Y.-Z.; Liu, H.; Zhang, G.; Lu, J.; Jiang, H.-L.; $\mathrm{Xu}, \mathrm{H}$. Hollow Metal-Organic Framework Nanospheres via Emulsion-Based Interfacial Synthesis and Their Application in Size-Selective Catalysis. ACS Appl. Mater. Interfaces 2014, 6, 18163-18171.

13. Yang, Z.; Li, Z.; Yang, Y.; Xu, Z.J. Optimization of $\mathrm{Zn}_{x} \mathrm{Fe}_{3-x} \mathrm{O} 4$ Hollow Spheres for Enhanced Microwave Attenuation. ACS Appl. Mater. Interfaces 2014, 6, 21911-21915.

14. Wang, M.; Zhang, W.; Wang, J.; Wexler, D.; Poynton, S.D.; Slade, R.C. T.; Liu, H.; Winther-Jensen, B.; Kerr, R.; Shi, D.; Chen, J. PdNi Hollow Nanoparticles for Improved Electrocatalytic Oxygen Reduction in Alkaline Environments. ACS Appl. Mater. Interfaces 2013, 5, 12708-12715.

15. Wang, L.; Imura, M.; Yamauchi, Y. Tailored Design of Architecturally Controlled Pt Nanoparticles with Huge Surface Areas toward Superior Unsupported Pt Electrocatalysts. ACS Appl. Mater. Interfaces 2012, 4, 2865-2869.

16. Chen, D.; Cui, P.; He, H.; Liu, H.; Yang, J. Highly Catalytic Hollow Palladium Nanoparticles Derived From Silver@silver-palladium Core-shell Nanostructures for the Oxidation of Formic Acid. J. Power Sources 2014, 272, 152-159.

17. Wu, P.; Wang, H.; Tang, Y.; Zhou, Y.; Lu, T. Three-Dimensional Interconnected Network of Graphene-Wrapped Porous Silicon Spheres: In Situ Magnesiothermic-Reduction Synthesis and Enhanced Lithium-Storage Capabilities. ACS Appl. Mater. Interfaces 2014, 6, 3546-3552.

18. Malolepszy, A.; Mazurkiewicz, M.; Mikolajczuk, A.; Stobinski, L.; Borodzinski, A; Mierzwa, B.; Lesiak, B.; Zemek, J.; Jiricek, P. Influence of Pd-Au/MWCNTs surface treatment on catalytic activity in the formic acid electrooxidation. Phys. Status Solidi C 2011, 8, 3195-3199. 
19. Qu, K.; Wu, L.; Ren, J.; Qu, X. Natural DNA-Modified Graphene/Pd Nanoparticles as Highly Active Catalyst for Formic Acid Electro-Oxidation and for the Suzuki Reaction. ACS Appl. Mater. Interfaces 2012, 4, 5001-5009.

20. Zhang, L.; Wan, L.; Ma, Y.; Chen, Y.; Zhou, Y.; Tang, Y.; Lu, T. Crystalline Palladium-cobalt Alloy Nanoassemblies with Enhanced Activity and Stability for The Formic Acid Oxidation Reaction. Appl. Catal. B 2013, 229- 235.

21. 21. Zhang, L.; Sui, Q.; Tang, T.; Chen, Y.; Zhou, Y.; Tang, Y.; Lu, T. Surfactant-free palladium nanodendrite assemblies with enhanced electrocatalytic performance for formic acid oxidation. Electrochem. Commun. 2013, 32, 43-46.

22. Ott, A.; Yu, X.; Hartmann, R.; Rejman, J.; Schütz, A.; Ochs, M.; J. Parak, W.; Carregal-Romero, S. Light-Addressable and Degradable Silica Capsules for Delivery of Molecular Cargo to the Cytosol of Cells.Chem. Mater. 2015, doi:10.1021/cm502472g.

23. Dahlberg, K.A.; Schwank, J.W. Synthesis of $\mathrm{Ni@SiO} 2$ Nanotube Particles in a Water-in-Oil Microemulsion Template. Chem. Mater. 2012, 24, 2635-2644.

24. Kim, S.M.; Jeon, M.; Kim, K.W.; Park, J.; Lee, I.S. Postsynthetic Functionalization of a Hollow Silica Nanoreactor with Manganese Oxide-Immobilized Metal Nanocrystals Inside the Cavity. $J$. Am. Chem. Soc. 2013, 135, 15714-15717.

25. Deng, T.-S.; Marlow, F. Synthesis of Monodisperse Polystyrene@Vinyl-SiO 2 Core-Shell Particles and Hollow $\mathrm{SiO}_{2}$ Spheres. Chem. Mater. 2011, 24, 536-542.

(C) 2015 by the authors; licensee MDPI, Basel, Switzerland. This article is an open access article distributed under the terms and conditions of the Creative Commons Attribution license (http://creativecommons.org/licenses/by/4.0/). 